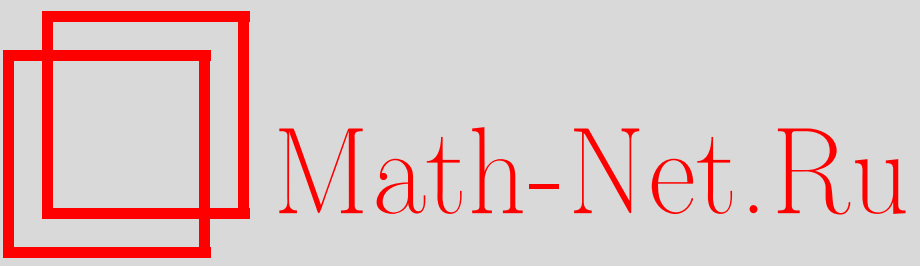

Н. А. Вешев, Вырождение решений уравнения Гойна при слиянии особенностей, TMФ, 1997, том 110, номер 2, 228-232

DOI: https://doi.org/10.4213/tmf963

Использование Общероссийского математического портала Math-Net.Ru подразумевает, что вы прочитали и согласны с пользовательским соглашением

http://www.mathnet.ru/rus/agreement

Параметры загрузки:

IP : 18.209 .158 .208

26 апреля 2023 г., 17:05:16 


\section{ВЫРОЖДЕНИЕ РЕШЕНИЙ УРАВНЕНИЯ ГОЙНА ПРИ СЛИЯНИИ ОСОБЕННОСТЕЙ}

Рассматриваются предельные переходы в процессе вырождения уравнения Гойна. Показано, что собственные функции уравнения Гойна переходят в собственные функции вырожденного уравнения Гойна.

\section{1. ВВЕДЕНИЕ}

Физические приложения уравнения Гойна и его вырожденных случаев $[1,2]$ объясняют большой интерес к этим уравнениям в последнее время $[3,4,5]$. Уравнение Гойна

$$
\frac{d^{2} y}{d z^{2}}+\left(\frac{\gamma}{z}+\frac{\delta}{z-1}+\frac{\varepsilon}{z-a}\right) \frac{d y}{d z}+\frac{\alpha \beta z-q}{z(z-1)(z-a)} y=0
$$

является уравнением с четырьмя особыми точками $0,1, a, \infty$. В работе используется понятие $s$-ранга, введенное в [4]. А именно, $s$-ранг регулярной особой точки, если она не является элементарной, равен 1 . Ранг конечной иррегулярной особой точки есть наибольшее из чисел - кратности полюса коэффициента при производной в уравнении вида (1) и половины кратности полюса коэффишиента при $y$. В уравнении Гойна каждая из особых точек имеет $s$-ранг равный 1 . Формальное слияние особенностей (например, когда $a \rightarrow \infty$ ) приводит к тому, что число параметров уравнения уменьшается более чем на 1. Подобньй процесс называется слабым вырождением. При этом суммарный $s$-ранг не сохраняется, и в результате получается уравнение гипергеометрического типа. В данной статье рассматривается другой процесс вырождения, сохраняющий сумму $s$-рангов особых точек.

Для получения вырожденного уравнения Гойна с теми же характеристиками в особых точках

$$
\frac{d^{2} y}{d z^{2}}+\left(\frac{\gamma}{z}+\frac{\delta}{z-1}+p\right) \frac{d y}{d z}+\frac{\alpha p z-q}{z(z-1)} y=0
$$

нужно, чтобы при стремлении $a$ к бесконечности и другие параметры уравнения (1) стремились к бесконечности. Пусть

$$
\beta=-p \alpha, \quad q=-a q, \quad \varepsilon=-p a+\alpha+1-\delta-\gamma .
$$

* Санкт-Петербургская государственная академия аэрокосмического приборостроения 
ВЫРОЖДЕНИЕ РЕШЕНИЙ УРАВНЕНИЯ ГОЙНА ПРИ СЛИЯНИИ ОСОБЕННОСТЕЙ 229

При этом число свободных параметров уменьшается на 1 , а получаюшееся уравнение (2) имеет в особых точках совокупность $s$-рангов $\{1,1,2\}$, т. е. в точках $z=0, z=1$ характер особенности сохраняется, а при $z=\infty$ особая точка становится иррегулярной.

В работах $[3,4]$ было показано, что при таком переходе сохраняются интегральные соотношения для специальных функций - решений уравнений (1) и (2). Однако при этом остается открытым вопрос о том, переходят ли при этом собственные функции уравнения (1) в собственные функции уравнения (2) [5].

Решение этого вопроса сушественно зависит от области изменения независимой переменной $z$. Для интервала $(0,1)$ удаление особенности $a \rightarrow \infty$ происходит "вдали" от него, в то время как переход собственных функций уравнения $(1)$ на интервале $(1, a)$ в собственные функции уравнения $(2)$ на $(1, \infty)$ затрагивает точку слияния особенностей $z=\infty$.

\section{2. ПЕРЕХОД ОТ УРАВНЕНИЯ ГОЙНА К ВЫРОЖДЕННОМУ УРАВНЕНИЮ}

Рассмотрим первый случай, когда $z \in(0,1)$. Вводя операторы

$$
\begin{aligned}
& \Lambda_{1}=z(z-1) \frac{d^{2}}{d z^{2}}+(\gamma(z-1)+\delta z) \frac{d}{d z}, \\
& \Lambda_{2}=z(z-1) \frac{d}{d z},
\end{aligned}
$$

можно записать уравнения (1) и (2) в виде

$$
\begin{aligned}
& L y=(z-a) \Lambda_{1} y+\varepsilon \Lambda_{2} y+(\alpha \beta z-q) y=0, \\
& \widehat{L} y=\Lambda_{1} y+p \Lambda_{2} y+(\alpha p z-q) y=0 .
\end{aligned}
$$

В обоих случаях, следуя $[6,7]$, произвольные решения (4) и (5) ишутся в виде

$$
y=\sum_{m=-\infty}^{\infty} c_{m} y_{\nu_{0}+m}
$$

где $y_{\nu}$ - решение гипергеометрического уравнения

$$
\Lambda_{1} y_{\nu}=\nu(\nu+\omega) y_{\nu}, \quad y_{\nu}=F(-\nu, \nu+\omega, \gamma, z) .
$$

Прежде чем подставлять представление (6) в (4) и (5), заметим, что

$$
\begin{aligned}
z y_{\nu} & =A_{\nu} y_{\nu+1}+B_{\nu} y_{\nu}+C_{\nu} y_{\nu-1}, \\
\Lambda_{2} y_{\nu} & =A_{\nu}^{\prime} y_{\nu+1}+B_{\nu}^{\prime} y_{\nu}+C_{\nu}^{\prime} y_{\nu-1},
\end{aligned}
$$

где

$$
\begin{array}{ll}
A_{\nu}=-\frac{(\nu+\omega)(\nu+\gamma)}{(2 \nu+\omega)(2 \nu+\omega+1)}, & A_{\nu}^{\prime}=-\frac{\nu(\nu+\omega)(\nu+\gamma)}{(2 \nu+\omega)(2 \nu+\omega+1)}, \\
B_{\nu}=\frac{2 \nu(\nu+\omega)+\gamma(\omega-1)}{(2 \nu+\omega-1)(2 \nu+\omega+1)}, & B_{\nu}^{\prime}=\frac{\nu(\nu+\omega)(\nu+\delta-1)}{(2 \nu+\omega)(2 \nu+\omega-1)} \\
C_{\nu}=-\frac{\nu(\nu+\delta-1)}{(2 \nu+\omega)(2 \nu+\omega-1)}, & C_{\nu}^{\prime}=-\frac{\nu(\nu+\omega)(\nu+\delta-1)}{(2 \nu+\omega)(2 \nu+\omega-1)}
\end{array}
$$


В этих обозначениях операторы $L$ и $\widehat{L}$ можно записать следующим образом:

$$
\begin{aligned}
& L y_{\nu}=D_{\nu} y_{\nu-1}+E_{\nu} y_{\nu}+F_{\nu} y_{\nu+1}, \\
& \widehat{L} y_{\nu}=D_{\nu}^{\prime} y_{\nu-1}+E_{\nu}^{\prime} y_{\nu}+F_{\nu}^{\prime} y_{\nu+1}
\end{aligned}
$$

где

$$
\begin{aligned}
D_{\nu}= & -\frac{\nu(\nu+\omega-\alpha)(\nu+\omega-\beta)(\nu+\delta-1)}{(2 \nu+\omega)(2 \nu+\omega-1)} \\
E_{\nu}= & \frac{\varepsilon \nu(\nu+\omega)(\nu-\delta)+(\nu(\nu+\omega)+\alpha \beta)(2 \nu(\nu+\omega)+\gamma(\omega-1))}{(2 \nu+\omega+1)(2 \nu+\omega-1)}- \\
& -a \nu(\nu+\omega)-q \\
F_{\nu}= & -\frac{(\nu+\alpha)(\nu+\beta)(\nu+\omega)(\nu+\gamma)}{(2 \nu+\omega)(2 \nu+\omega-1)}, \\
D_{\nu}^{\prime}= & p\left(C_{\nu}^{\prime}+\alpha C_{\nu}\right), \\
E_{\nu}^{\prime}= & \nu(\nu+\omega)+p\left(B_{\nu}^{\prime}+\alpha B_{\nu}\right)-q, \\
F_{\nu}^{\prime}= & p\left(A_{\nu}^{\prime}+\alpha A_{\nu}\right) .
\end{aligned}
$$

Подстановка решения $y$ в виде (6) в уравнения (4) и (5) приводит к бесконечной системе уравнений на коэффициенты $c_{r}$ :

$$
P_{r} c_{r-1}+S_{r} c_{r}+R_{r} c_{r+1}=0
$$

где

$$
P_{r}=F_{\nu_{0}+r-1}, \quad S_{r}=E_{\nu_{0}+r}, \quad R_{r}=D_{\nu_{0}+r+1}
$$

для уравнения Гойна (4), и

$$
P_{r}^{\prime} c_{r-1}+S_{r}^{\prime} c_{r}+R_{r}^{\prime} c_{r+1}=0
$$

где

$$
P_{r}^{\prime}=F_{\nu_{0}+r-1}^{\prime}, \quad S_{r}^{\prime}=E_{\nu_{0}+r}^{\prime}, \quad R_{r}^{\prime}=D_{\nu_{0}+r+1}^{\prime}
$$

для вырожденного уравнения Гойна (5). Легко установить, что при выполнении условий (3)

$$
\lim _{a \rightarrow \infty} P_{r}=P_{r}^{\prime}, \quad \lim _{a \rightarrow \infty} S_{r}=S_{r}^{\prime}, \quad \lim _{a \rightarrow \infty} R_{r}=R_{r}^{\prime} .
$$

Таким образом, бесконечные системы для решений уравнений (4) и (5) переходят друг в друга. Однако этого факта мало для доказательства перехода решений уравнений друг в друга.

Если раньше речь шла о произвольных решениях, то теперь перейдем к специальным решениям - собственным функциям.

ТЕОРема. Если $y_{1}(z, a)$ есть собственная функция уравнения (1) на промехутке $(0,1)$, отвечающая собственному числу $q$, а $y_{2}(z)$ - собственная функция (2), отвечающая собственному числу, получаемому из q в соответствии $c(3)$, то при выполнении (3)

$$
\lim _{a \rightarrow \infty} y_{1}(z, a)=y_{2}(z) .
$$


ДокАЗАТЕЛЬСТво. Известно [6, 7], что если акцессорньй параметр $q$ принимает одно из собственных значений, то в ряду (6) суммирование будет происходить от $m=0$. В соответствии с (7) условие на обрыв суммирования при $m=0$ (т. е. дисперсионное уравнение) записывается так:

$$
\frac{S_{0}(q, a)}{R_{0}}=A(q, a)
$$

где $A(q, a)$ можно представить бесконечной непрерывной дробью

$$
A(q, a)=-\frac{P_{1}}{S_{1}-R_{1} \frac{P_{2}}{S_{2}-\ldots}} .
$$

Известно [7], что эта дробь сходится, если корни уравнения

$$
\rho^{2}+2(2 a-1) \rho+1=0
$$

различаются по модулю. Дисперсионное уравнение является трансцендентным уравнением относительно $q$. При $a \rightarrow \infty$ и выполнении условий (3) уравнение (8) переходит в дисперсионное уравнение для вырожденных функций Гойна

$$
\frac{S_{0}^{\prime}(a)}{R_{0}^{\prime}}=A^{\prime}(a)
$$

где $A^{\prime}(a)$ - соответствуюшая непрерывная дробь для вырожденного уравнения. При этом вследствие равномерной по $a$ сходимости непрерывной дроби [7] величина $A(q, a)$ переходит в $A^{\prime}(a)$. Из-за непрерывности функций пределы собственных значений уравнения (1) равны собственным значениям уравнения (2).

Область сходимости ряда (6) для уравнения (1) определяется условием

$$
\left|\frac{1+Z}{1-Z}\right|<\left|\frac{1}{\rho}\right|
$$

где $Z=\sqrt{1-z^{-1}}, \rho$ - наименьший по абсолютной величине корень уравнения (9).

При $a \rightarrow \infty$ область сходимости ряда увеличивается и стремится ко всей комплексной плоскости. Следовательно, в окрестности промежутка $(0,1)$ имеет место равномерная сходимость ряда (6), и можно утверждать, что собственные функции уравнения Гойна стремятся к собственным функциям вырожденного уравнения Гойна.

Перейдем теперь к рассмотрению собственных функций уравнения Гойна на $(1, \infty)$. При дробно-линейном преобразовании $t=\frac{z-1}{a-1}$ уравнение Гойна перейдет само в себя, только с другими параметрами:

$$
\gamma_{n}=\frac{\gamma}{a-1}, \quad q_{n}=\frac{q-\alpha \beta}{a-1}, \quad a_{n}=\frac{1}{1-a}
$$


и с особенностями в точках $0, a_{n}, 1, \infty$. При этом интервал $(1, \infty)$ отобразится в интервал $(0,1)$, т.е. можно будет воспользоваться результатами предыдущего случая. Однако вопрос о сходимости ряда теперь решается с помощью более тонких оценок, т. к.

$$
\lim _{a \rightarrow \infty} \frac{c_{m+1} y_{\nu_{0}+m+1}}{c_{m} y_{\nu_{0}+m}}=1
$$

При этом получаем для больших номеров $\nu$ и $a \rightarrow \infty$

$$
\begin{aligned}
D_{\nu} & =-\nu^{2}+\nu(\gamma-\alpha)+O(1), \\
F_{\nu} & =-\nu^{2}+\nu(-\alpha-\delta)+O(1), \\
E_{\nu} & =2 \nu^{2}+\nu(-\gamma+2 \alpha+\delta+p)+O(1),
\end{aligned}
$$

и, значит, при $r \rightarrow \infty$ имеем $c_{r}=O(\exp ( \pm \sqrt{p r}))$. Знак "-" соответствует меньшему по модулю корню уравнения (8), т. е. случаю, когда $q$ равняется одному из собственных значений.При этом $y_{r}=O\left(r^{1 / 4-\delta / 2}\right)$. Таким образом, ряд (6) опять оказывается равномерно (по $a$ ) сходяшимся при конечных $z$, и можно утверждать, что собственные функции уравнения Гойна на $(1, a)$ переходят в собственные функции вырожденного уравнения Гойна на $(1, \infty)$.

Автор выражает благодарность С.Ю.Славянову за постановку задачи и ряд ценных замечаний. Статья написана при финансовой поддержке фонда Сороса (грант R5X000).

\section{Список литературы}

[1] И.В.Комаров, Л.И.Пономарев, С.Ю.Славянов. Сфероидальные и кулоновские сфероидальные функции. М.: Наука, 1976.

[2] S. Chandrasekhar. The Mathematical Theory of Black Holes. Oxford-New-York: Oxford University Press, 1983.

[3] А.Я.Казаков, С.Ю.Славянов. ТМФ. 1996. Т. 107. С. 388.

[4] А.Зеегер, В.Лай, С.Ю.Славянов. ТМФ. 1995. Т. 104. С. 233.

[5] В. Лай. ТМФ. 1994. Т. 101. С. 360.

[6] A.Ronveaux. Heun's differential equation. Oxford: Oxford University Press, 1995.

[7] Г.Бейтмен, А.Эрдейи. Высшие трансцендентные функции. М.: Наука, 1967.

Поступила в редакцию 23.I.1996 г., после доработки 8.Х.1996 г.

\section{N.A. Veshev \\ DEGENERATION OF SOLUTIONS OF HEUN'S EQUATION UNDER FUSION OF SINGULARITIES}

Degenerations of Heun's equation are considered. It is shown that the eigenvalues of Heun's equation get over into the ones of the degenerate equation. 